\title{
Situación Actual de la Psicología Educativa en Europa
}

\section{Current State of Educational Psychology in Europe}

\author{
Leopold Carreras Truñó* \\ Ámbito privado, España
}

\begin{abstract}
Resumen. Con este artículo se pretende dar a conocer los principales problemas surgidos y los objetivos conseguidos estos últimos años con respecto a las normativas de los diferentes países europeos referentes a la función y perfil del psicólogo educativo, así como las conclusiones del Proyecto ESPIL (European School Psychologists Improve Lifelong Learning), presentado a la Unión Europea (UE) el pasado mes de octubre de 2010, y con el cual se ha intentado sensibilizar sobre el impacto de la evolución de las políticas de los países europeos en materia de educación y formación de los profesionales de la psicología educativa durante la última década, así como alentar a las asociaciones miembros de la UE a estudiar las consecuencias de estas políticas de educación, formación y prestación de servicios de psicólogos educativos, incluidos los perfiles profesionales, competencias y cualificaciones dentro de los nuevos paradigmas de formación permanente.

Palabras clave: formación continuada, psicólogo educativo, orientador, perfil.
\end{abstract}

\begin{abstract}
This article aims to set out the main problems and objectives achieved in recent years with regard to regulation in the various European countries of the role and profile of the educational psychologist. It also sets out the findings of the ESPIL Project (European School Psychologists Improve Lifelong Learning) submitted to the European Union (EU) last October 2010. This project has attempted to raise awareness of the impact of policy developments in European countries in the education and training of educational psychology professionals in the last decade, and encourage EU member associations to consider the consequences of these policies on education, training and provision of educational psychologists, including job profiles, competencies and qualifications within the new paradigms of lifelong learning.

Keywords: lifelong learning, educational psychologist, counselor, profile.
\end{abstract}

\section{Introducción}

Ante la confusión generalizada que observamos que se da sobre quién respalda a los psicólogos educativos y desde qué punto de vista lo hace, hemos considerado necesario llevar a cabo una breve introducción aclaratoria sobre los diferentes y más importantes organismos que trabajan en la actualidad en el ámbito nacional e internacional, a fin de intentar clarificar un poco el panorama existente.

En primer lugar, tenemos la Asociación Internacional de Psicología Escolar ISPA (International School Psychology Association). Como su nombre indica, esta asociación abarca a los psicólogos escolares de todo el mundo que, sobre todo, son socios que participan de manera individual, aunque también se acepta en su seno a las asociaciones que deseen formar parte de ella. El término psicólogo escolar se usa de manera general para referirse a «aquellos profesionales preparados en psicología y educación que están reconocidos como especia-

* Psicólogo. Representante en España de la Network of European Psychologists in the Educational System (NEPES), Asesor del Área de Psicología Educativa del Consejo General de Colegios Oficiales de Psicólogos de España, Vocal de la Junta Directiva de la Sección Educativa del Colegio Oficial de Psicólogos de Cataluña (COPC), Asesor del Consejo Social del COPC.

La correspondencia sobre este artículo debe enviarse al autor al email: leopold.carreras@gmail.com listas en la provisión de servicios psicológicos a niños y jóvenes en el contexto de las escuelas, familias y otros entornos que puedan incidir en su crecimiento y desarrollo». Aunque siguen usando el término psicólogo escolar, la directiva de ISPA y prácticamente todos sus asociados están de acuerdo en que es mucho más acertado y exacto usar psicólogo educativo, pues su radio de acción -como podemos ver por la definición que usanno se lleva a cabo exclusivamente en las escuelas, pero siguen usando el término antiguo debido a que en Estados Unidos sí existe diferencia entre el trabajo y la formación de un psicólogo educativo y la de un psicólogo escolar. Aunque los términos «psicología educativa» y «psicología escolar» son frecuentemente utilizados como sinónimos, los teóricos e investigadores de Norteamérica prefieren ser identificados como psicólogos educacionales, mientras que los profesionales que desempeñan específicamente sus labores en escuelas o en tareas relacionadas con la escuela se identifican allí como psicólogos escolares. Aunque ISPA sea un organismo internacional, fue fundado en Estados Unidos. De ahí que, para evitar los problemas que significaría introducir un cambio en el nombre de una asociación histórica como ésta, pues lleva en funcionamiento desde principios de los años setenta, se haya decidido continuar con este término. En la actualidad, el Consejo General de Colegios Oficiales de Psicólogos de España (CGCOP) se ha hecho miembro de ISPA. 
En segundo lugar, existe la Asociación Nacional de Psicólogos Escolares NASP (National Association of School Psychologists), en la que sus miembros son exclusivamente personas individuales (psicólogos), pues no aceptan a asociaciones. A pesar de que su radio de acción es exclusivamente para Norteamérica (EE.UU. y Canadá), hemos creído necesario incluirlo en este resumen debido a la enorme influencia que ejerce sobre el resto de asociaciones internacionales. Para la NASP, los psicólogos escolares son «aquellos que ayudan a los niños y jóvenes a tener éxito académico, social, conductual y emocional. Colaboran con los educadores, padres y otros profesionales para crear ambientes de aprendizaje seguro, saludable y de apoyo, que fortalezcan las conexiones entre el hogar, la escuela y la comunidad para todos los estudiantes. Los psicólogos escolares son profesionales altamente preparados tanto en psicología como en educación, y han debido completar como poco un programa de especialidad (por lo menos sesenta horas semestrales de posgrado) que incluya una práctica supervisada de un mínimo de un año de duración. Esta formación hace hincapié en la preparación en salud mental e intervenciones educativas, desarrollo del niño, aprendizaje, conducta, motivación, currículo e instrucción, evaluación, consulta, colaboración, leyes educativas y sistemas». Como podemos apreciar, esta definición abarca y amplía un poco la ofrecida por ISPA, en el sentido de que especifica la formación que debe tener el psicólogo escolar. Y, lógicamente, dado que su ámbito de actuación es Norteamérica, también ellos mantienen el término «psicólogo escolar» por los mismos motivos que ISPA.

\section{Situación en Europa}

En Europa, tenemos la Federación Europea de Asociaciones de Psicólogos o EFPA (European Federation of Psychologists' Associations), dentro de la cual existe un Grupo de Trabajo (Task Force) llamado NEPES (Network of European Psychologists in the Educational System o Red de Psicólogos Europeos en el Sistema Educativo), que desde este pasado verano de 2011 ha pasado a ser ya una Comisión Permanente (Standing Committee). EFPA, al contrario que la NASP, sólo admite en su seno a asociaciones nacionales de psicología y no a miembros individuales. En España, quien ejerce esta función es el Consejo General de Colegios Oficiales de Psicólogos de España (CGCOP). Para EFPA, el psicólogo en el sistema educativo es un «psicólogo profesional con la licenciatura (grado) de psicología, un máster en psicología educativa y experiencia en el campo de la educación (EFPA, 2001)». Posteriormente, NEPES añadió que «los psicólogos en el sistema educativo tienen una posición clave como puente de unión entre el sector de la salud mental, el sector educativo y el sector social» (NEPES, 2010).
Los principales servicios que se ofrecen desde NEPES son, en primer lugar, presentar todo el amplio campo de conocimientos de la psicología educativa, las bases de ésta, así como todas las experiencias, estudios, etc., de ámbito europeo, de manera que esté disponible para cualquier persona interesada; en segundo lugar, se pretende clarificar y fortalecer el papel del psicólogo de la educación en Europa y su diferenciación en relación con otros profesionales que trabajan en la misma área (pedagogos, psicopedagogos, etc.); en tercer lugar, promover el intercambio profesional entre los psicólogos educativos europeos; en cuarto lugar, promover el uso de la psicología como medio para mejorar el bienestar de todos aquellos a quienes estos psicólogos ofrecen sus servicios; en quinto lugar, promover los derechos de los niños, y, por último, aumentar los contactos con los políticos y los organismos pertinentes a nivel tanto regional como europeo.

Por consiguiente, NEPES es el organismo que nos representa a quienes nos dedicamos a la psicología educativa en Europa. Sin embargo, debemos tener presente que dentro del CGCOP (Consejo General de Colegios Oficiales de Psicólogos de España) también existe el Área de Psicología Educativa, que en un futuro -esperemos que cercano- pasará a convertirse en División de Psicología Educativa, pues sus estatutos ya están aprobados por la Junta, y éste es el organismo que velará por el futuro de nuestra profesión en España. Del mismo modo, muchos de los Colegios Oficiales de Psicólogos que existen en España poseen en su estructura una Sección de Psicología Educativa, que es precisamente la entidad a la que en primer lugar deberemos dirigirnos para resolver cualquier duda, consulta, etc., que como psicólogos educativos tengamos.

Como hemos podido apreciar, las definiciones que se están usando en la actualidad en el ámbito internacional difieren muy poco entre ellas, siendo complementarias unas de otras. En todas se remarca la necesidad de una preparación específica y, a continuación, veremos có-mo, en España, la opinión generalizada también va en la misma dirección.

\section{Situación en España}

Si hay una fecha que quienes nos dedicamos a esto de la psicología educativa deberíamos recordar, es el 31 de enero de 2009. Ese día, y en el marco del I Encuentro Nacional de Profesionales de la Psicología de la Educación, organizado por el Área de Psicología Educativa del CGCOP, y llevado a cabo en la entonces recién estrenada sede central del COPC (Colegio Oficial de Psicólogos de Cataluña), se establecieron las bases para el futuro de nuestra profesión en España en torno a cuatro grandes áreas: formación básica, trabajo en los institutos y equipos, formación continua y formación de posgrado. En ese encuentro se definió al psicólogo de la educación como «el profesional de la 
psicología que tiene por trabajo la reflexión e intervención sobre el comportamiento humano en situaciones educativas, mediante el desarrollo de las capacidades de las personas, grupos e instituciones. Se entiende el término educativo en el sentido más amplio (situaciones formales e informales), por lo que se recomienda dejar de usar el término psicología escolar para referirse a la psicología educativa, ya que la primera únicamente hace referencia a la psicología educativa en contextos escolares» (Infocop, 2009). Como apreciaremos, esta definición también sigue en la misma línea de las ofrecidas por el resto de organismos internacionales citados anteriormente.

En ese encuentro se intentó analizar el porqué del deterioro progresivo de la imagen del psicólogo educativo, fundamentalmente dentro del ámbito escolar, y la respuesta que se dio en ese momento se orientaba hacia tres motivos principales: $a$ ) el auge de la psicopedagogía en el ámbito académico y los psicopedagogos, con una limitada formación psicológica; $b$ ) la identificación errónea entre psicólogo educativo y orientador, y c) el propio deterioro en el ejercicio de su profesión, sobre todo -añadiríamos- debido al hecho de que, a pesar de que el psicólogo es una figura socialmente respetada y demandada, con un perfil profesional y unas competencias profesionales reconocidas, las distintas administraciones educativas han ido diluyendo esta figura, equiparándola a la de profesionales de otras titulaciones y con una formación claramente distinta de la del psicólogo educativo, obligándonos a trabajar muchas veces más como un profesor de cualquier asignatura (matemáticas, inglés, etc.) que como psicólogos propiamente dichos, con nuestros roles y perfil profesional claramente delimitados.

\section{¿Perfil claramente delimitado?}

Podría parecer que no está tan claro el perfil que debe tener un psicólogo educativo; incluso a lo largo de los últimos quince años se han hecho diversas «Jornadas sobre el rol, el perfil y las funciones del "profesor" de psicología y pedagogía de la enseñanza secundaria» en Barcelona precisamente para discutir este asunto. Pero este dilema no sólo se ha dado en España, sino también en toda Europa, pues la situación laboral de los psicólogos educativos estaba (y está) siendo cuestionada -en el sentido de re-perfilada- en toda la Comunidad Europea. Países como Suecia, Alemania, Dinamarca, Eslovaquia, Chequia, Lituania, Malta, Luxemburgo o Reino Unido, entre otros, ya se nos han adelantado en lo referente a crear leyes educativas con las necesidades y competencias específicas que debe atender el psicólogo en el sistema escolar, entre otros factores, gracias a la intervención y presión llevada a cabo desde NEPES (EFPA) en alguno de esos países, mientras que en España todavía no ha surgido una ley que delimite de manera clara este perfil, a pesar de todos los cambios que han sufrido las leyes educativas últimamente.

Por otro lado, sí es cierto que, poco a poco, parece que algo se está moviendo, pues el pasado mes de marzo de 2010, el Consejo General de Colegios Oficiales de Psicólogos (CGCOP) junto con la Conferencia de Decanos de Psicología de las Universidades Españolas (CDPUE) elaboró un informe sobre la psicología educativa en el sistema educativo español para presentar al Ministerio de Educación a fin de que se añadiera al «Pacto social y político por la educación». En este informe se analizaban las necesidades del sistema educativo y la situación actual de la psicología educativa dentro del sistema, con la finalidad de definir adecuadamente el papel y las funciones del psicólogo educativo. Posteriormente, el pasado mes de septiembre de 2010 se llegó a un «Acuerdo de inserción de los psicólogos educativos en los centros de enseñanza no universitaria», que es un acuerdo en defensa de la cualificación profesional y de las iniciativas parlamentarias en la mejora de la calidad educativa por parte de algunos de los máximos representantes del mundo educativo, como son la Federación de Asociaciones de Directivos de Centros Educativos Públicos (FEDADI), la Confederación Española de Asociaciones de Padres y Madres de Alumnos (CEAPA), la Confederación Católica Nacional de Padres de Familia y de Padres de Alumnos (CONCAPA), el Consejo General de Colegios Oficiales de Psicólogos (CGCOP) y la Conferencia de Decanos de Psicología de las Universidades Españolas (CDPUE), quienes pusieron de manifiesto públicamente que existían unas necesidades sentidas y expresadas dentro de los centros educativos no universitarios españoles que requerían, para su adecuada solución, el ejercicio de profesionales no docentes especialmente formados, siendo uno de estos profesionales el psicólogo educativo: licenciado o graduado en psicología y con un máster específico en psicología de la educación (aspecto que se pretende conseguir en un futuro no muy lejano).

En el mes de mayo de 2011, se presentó una Proposición No de Ley en la que se instaba al Gobierno a la inserción del psicólogo educativo en la organización de los centros escolares. Sin embargo, esta proposición finalmente fue denegada por escasos votos de diferencia, pues la gran mayoría de partidos políticos la avalaron excepto el mayoritario en el Gobierno, por lo que nuestra «lucha» en la consecución de esta importante -vital- meta para la subsistencia en adecuadas condiciones de nuestro puesto de trabajo como psicólogos educativos debe continuar. De hecho, el actual Área de Psicología Educativa del CGCOP ya tiene previstas varias reuniones para explorar y determinar cómo continuar trabajando para conseguir nuestros lícitos objetivos.

Pues bien, retomando el hilo inicial de este capítulo, todas estas leyes de diferentes países están agrupadas 
en torno a ciertos aspectos básicos, siendo el principal de ellos el considerar al psicólogo educativo como un profesional clave dentro de los sistemas educativos de cada país a fin de conseguir una mejora en la calidad de dichos sistemas. Para conseguir este objetivo, cada país ha creado sus propios modelos sobre el perfil que deben poseer estos psicólogos, aunque también cabe comentar que, en líneas generales, este perfil coincide en la mayoría de países -y también en los propuestos por el CGCOP y la CDPUE-, siendo los objetivos básicos que debe tener un psicólogo educativo los siguientes:

- Mejorar la calidad del aprendizaje de los alumnos/as, tanto de preescolar como de primaria y secundaria.

- Diagnosticar e intervenir ante cualquier tipo de trastorno (emocional, conductual, de personalidad, etc.), que pueda afectar a un alumno/a en su salud o proceso de aprendizaje.

- Prevenir, detectar y evaluar cualquier tipo de problema relacionado con la educación que sea susceptible de mejorarse mediante la intervención psicológica, tanto personal como grupal o institucional.

- Contribuir a la inclusión de alumnos/as con algún grado de minusvalía.

- Participar en la orientación profesional de los alumnos/as de secundaria.

- Participar en la organización y funcionamiento de los aspectos de la vida escolar relacionados con proyectos educativos.

- Asesoramiento a padres, profesores, alumnos y personal escolar y comunitario.

- Investigación relacionada con aspectos educativos.

- Mediar en los conflictos escolares (personales, grupales o institucionales).

- Intervención en situaciones de crisis.

Como veremos a continuación, estos aspectos son muy parecidos a los que se exponen en el citado informe sobre la psicología educativa en el sistema educativo español (CGCOP y CDPUE: «Propuestas para un Pacto Social y Político por la Educación. Ministerio de Educación»), que indica que el psicólogo educativo debería llevar a cabo, como aspectos fundamentales dentro del sistema educativo, las tareas de detección, evaluación, diagnóstico, asesoramiento e intervención tanto con alumnos como con familias, profesores e instituciones. Y continúa diciendo: «El psicólogo educativo debe ser capaz de realizar, sin perjuicio de las que lleven a cabo otros profesionales, las funciones de:

a) Asesoramiento a individuos, grupos e instituciones a partir de sus conocimientos especializados en psicología.

b) Mediación entre contextos, instituciones, grupos o personas para facilitar acuerdos.

c) Orientación de la persona a lo largo de su ciclo vital en aspectos psicológicos, conductuales y profesionales. d) Intervención en diferentes contextos: escolar, familiar, comunitario, etc.

e) Recopilación, análisis y valoración de la información relevante sobre los diversos elementos que intervienen en el proceso de enseñanza/ aprendizaje.

f) Evaluación y diagnóstico psicológico, elaboración de informes y orientaciones o propuestas de intervención.

g) Prevención y detección precoz de los problemas de salud mental, su derivación y seguimiento.

h) Mediación para la modificación de aquellas circunstancias del contexto generadoras de problemas, así como para dotar a los individuos de las competencias adecuadas para que puedan afrontar con éxito situaciones problemáticas.

i) Intervención desde un enfoque sistémico o global desde el que se aborda al sujeto en relación con su contexto socio-familiar y escolar.

j) Colaboración en el proceso y promoción del desarrollo integral del potencial de cada persona.

k) Diseño, planificación y selección de técnicas y recursos de intervención e investigación.

l) Respuesta a las demandas de las instituciones y de las administraciones educativas relativas a informes y dictámenes, especialmente a aquellos referidos al alumnado con necesidades educativas especiales.

m) Colaboración, dentro de sus conocimientos específicos, en intervenciones organizativas orientadas a la evaluación y la mejora de la calidad educativa.»

Posteriormente, el informe profundiza más, exponiendo que «en un ámbito más específico», el psicólogo educativo está en condiciones de:

1. Realizar la evaluación psicológica del sujeto, utilizando los instrumentos y técnicas psicológicas específicas, así como la elaboración del correspondiente informe psicológico.

2. Hacer una detección temprana y el diagnóstico de trastornos del desarrollo.

3. Diagnosticar:

- Discapacidades psíquicas: retraso mental y retraso madurativo.

- Altas capacidades intelectuales.

- Trastornos del comportamiento y de las emociones.

- Trastornos específicos del aprendizaje.

4. Hacer una intervención psicológica con los alumnos que presentan discapacidades sensoriales y motrices que lo precisen.

5. Realizar una intervención psicológica con alumnos o con grupos en situaciones de conflicto: violencia escolar, acoso escolar, conductas agresivas, etc.

6. Asesorar al profesorado para el manejo del grupo: resolución de conflictos, mejora de habilidades sociales, acoso escolar, etc. 
7. Asesorar al profesorado, a las familias y a los alumnos sobre los problemas y procesos psicológicos que afectan al aprendizaje: trastornos del sueño, trastornos de la alimentación, control de esfínteres y otros trastornos de conducta.

8. Asesorar a familias, a profesores y a alumnos que han estado expuestos a situaciones traumáticas, como fallecimiento de un familiar, malos tratos, abuso sexual, terrorismo, catástrofes, etc., junto a su posterior intervención y/o derivación.

El necesario ajuste de estas competencias al ámbito educativo aconseja que los profesionales que las desarrollen posean la formación básica en psicología y superen, así mismo, la formación especializada imprescindible que garantice la adecuación de sus conocimientos y de su ejercicio profesional.»

Ante esto, es evidente que para el ejercicio de estas funciones es necesaria la formación basada en la licenciatura/grado de psicología y una formación complementaria especializada de posgrado adquirida mediante un máster profesional en psicología de la educación que dote de las capacidades y competencias reseñadas, por lo que no podemos permitir que siga sucediendo como en la actualidad, que aquellas personas que poseen el máster de secundaria, al cual se puede acceder poseyendo una licenciatura de «cualquier» carrera, como derecho, económicas, filología, etc., lo cual recordemos que no capacita para las funciones citadas anteriormente, puede trabajar en los equipos de orientación y llevar a cabo, si se tercia, el rol de psicólogo educativo. Evidentemente, es diferente el caso de pedagogos y psicopedagogos, que sí pueden ejercer determinadas funciones dentro del equipo de orientación de las escuelas, pues para ello sí han estado preparados e instruidos, aunque con un rol, lógicamente, distinto del que debe realizar el psicólogo educativo.

Por tanto, para que todo ello se lleve a cabo de manera reglada en España, es necesario -como bien expusieron el CGCOP y la CDPUE- que el Ministerio de Educación tome cartas en el asunto y el citado «Pacto social y político por la educación» genere un desarrollo normativo, bien mediante el Estatuto del Funcionario Docente No Universitario, o bien mediante cualesquiera otras normas que se consideren oportunas, y se profesionalice de una vez por todas al psicólogo educativo, es decir, que adquiera la categoría profesional de «psicólogo» o «psicólogo educativo», aspecto que en la actualidad -como bien sabemostodavía no está regularizado.

\section{Europa}

\section{NEPES (Network of European Psychologists in the Educational System)}

NEPES, como se ha comentado anteriormente, es el acrónimo de Red de Psicólogos Europeos en el
Sistema Educativo. Se trata de una organización que ha crecido desde hace tan sólo cuatro años dentro de EFPA (European Federation of Psychologists Associations, o Federación Europea de Asociaciones de Psicólogos) y que, a pesar de su corta vida, son ya 25 los países europeos que han entrado a formar parte de ella. Su misión consiste en promocionar la psicología como una ciencia y una profesión dentro del sistema educativo.

Los principales servicios que se ofrecen son, en primer lugar, presentar todo el amplio campo de conocimientos de la psicología educativa, las bases de ésta, así como todas las experiencias, estudios, etc., de ámbito europeo, de manera que esté disponible para cualquier persona interesada; y, en segundo lugar, se pretende clarificar el papel del psicólogo de la educación en Europa y su diferenciación en relación con otros profesionales que trabajan en la misma área (pedagogos, psicopedagogos, etc.). Sin embargo, no se trata sólo de estos dos puntos principales, pues se abarca gran cantidad de áreas sumamente interesantes y prácticas, como:

- Promocionar la comunicación y cooperación entre psicólogos de la educación y sus organizaciones en el ámbito europeo.

- Ayudar a consolidar el estatus profesional y las condiciones de trabajo de los psicólogos que trabajan dentro del sistema educativo.

- Promover el acercamiento de los códigos éticos entre todos los psicólogos de Europa que trabajan en esta área.

- Promover el uso de la psicología como medio para mejorar el bienestar de todos aquellos a quienes estos psicólogos ofrecen sus servicios.

- Promover los Derechos de los Niños.

- Ayudar a todas las organizaciones miembros de NEPES a promover el interés por la psicología y los psicólogos dentro del sistema educativo de su propio país.

- Ser un referente para todas las instituciones europeas, gobiernos, así como de cualquier organización política, social o de marcada relevancia en el campo de la psicología educativa.

- Publicar estudios, investigaciones, prácticas, etc.

- Promover la reputación y el estatus de la psicología y los psicólogos que trabajan en el sistema educativo, así como la protección de sus intereses.

También es importante dar a conocer que a lo largo de los años 2008 a 2010 se ha llevado a cabo un proyecto sobre el tema de la formación continuada para los psicólogos educativos, así como para sentar las bases de cuál debe ser el perfil básico profesional del psicólogo de la educación en los países europeos y cómo mejorar la calidad de la educación/formación permanente que deben recibir estos psicólogos, todo lo cual conforma la base del proyecto ESPIL (European School Psychologists Improve Lifelong Learning), patrocinado por la Unión Europea y llevado a cabo por 
NEPES bajo el abrigo de EFPA (www.nepes.eu). Este proyecto lo han realizado psicólogos expertos en psicología de la educación de 22 países europeos, lo que ya de por sí indica la importancia que se está dando en la actualidad a nuestra profesión. Y vale la pena comentar que ya podemos confirmar que todos los datos de los que disponemos nos llevan a concluir que en toda Europa se están haciendo esfuerzos exactamente en la misma dirección que las propuestas del informe expuesto en el apartado previo -si es que éstas no se han llevado a cabo ya, como ha sucedido en los países anteriormente citados-.

La Resolución 2004 del Consejo de la UE sobre reforzar las políticas, sistemas y prácticas en materia de orientación permanente en Europa (Consejo de la Unión Europea, Bruselas, 18 de mayo de 2004, «Proyecto de Resolución del Consejo y de los Representantes de los Estados miembros reunidos en el seno del Consejo, sobre el fortalecimiento de las políticas, sistemas y prácticas en materia de orientación permanente en Europa», doc. núm.: 8448/04 EDUC 89 SOC 179), sitúa a la orientación en un nivel prioritario como un componente estratégico clave para la implementación de las estrategias de formación permanente (LLL o Lifelong Learning) a niveles regionales/nacionales. Los Psicólogos en el Sistema Educativo (PSE) trabajan en todo el ciclo vital de aprendizaje y proporcionan también orientación y asesoramiento, educación y capacitación del personal docente. NEPES surge -entre otros motivos- con el fin de apoyar las políticas de formación permanente en toda la UE para mejorar la organización de los PSE.

EFPA ha desarrollado desde el año 2001 el Certificado Europeo de Psicología (EuroPsy), financiado por la UE como una referencia de calidad de la UE para la educación de los psicólogos, que se aplica actualmente en varios Estados miembros de la UE, España incluido. Sin embargo, por el momento, su aplicación práctica se está llevando a cabo básicamente en el área de la psicología clínica. Ante este hecho, surgió la necesidad de comprobar si podría realizarse también en la psicología educativa. Por ello, EFPA ha pretendido que mediante el proyecto ESPIL:

- Se sensibilice desde NEPES sobre el impacto de la evolución de las políticas de la UE en materia de educación/formación durante la última década.

- Se aliente a sus asociaciones miembros a estudiar las consecuencias de estas políticas en la educación, capacitación y prestación de servicios de los PSE, incluyendo los perfiles profesionales, competencias y cualificaciones en los nuevos paradigmas de la formación permanente.

- Se lleve a cabo un desarrollo conjunto de recomendaciones de política sobre la base de los resultados del debate en torno a dos áreas importantes en la formación permanente:

1. La mejora de la calidad de la educación/formación del PSE.
2. Mejorar la calidad de la prestación de servicios en materia de formación permanente -se incluyen los puntos de vista de los interesados en la educación en este proceso-.

Este proyecto pretende preparar a las asociaciones nacionales de psicólogos educativos a desempeñar un papel proactivo en la aplicación de las recomendaciones de la UE en materia de educación y capacitación a nivel nacional. El proyecto ha dado lugar a un informe sobre el estado actual en Europa de la Psicología en el Sistema Educativo, incluyendo una declaración de EFPA en forma de documento oficial sobre su postura con respecto a las reformas en educación, formación, perfil profesional y la prestación de servicios del psicólogo educativo. El contenido de este documento se basa también en las presentaciones y los resultados de las dos conferencias ESPIL, la primera de enero de 2010 en Bruselas y la segunda de julio de 2010 en Dublín.

\section{Resumen del Proyecto ESPIL}

Toda la estructura de este proyecto se basa en tres afirmaciones, que anotamos a continuación, junto con una breve exposición de sus implicaciones.

\section{Afirmación 1. La disponibilidad de conocimientos psicológicos en los sistemas educativos representa una parte fundamental de la educación europea de calidad y de formación permanente.}

Las políticas de la UE sobre formación permanente (LLP o Lifelong Learning Policies) se apoyan desde todas las áreas de trabajo de los Psicólogos en el Sistema Educativo (PSE). Desde NEPES estamos de acuerdo en que los Servicios Psicológicos en las escuelas son importantes para:

- Promover la dimensión cualitativa de las LLP, incluyendo las competencias clave y competencias transversales, como la realización personal, la inclusión social, el empleo... En consecuencia, desde este punto de vista, la cuestión principal que debe hacerse sobre los PSE es: ¿existe un ambiente óptimo de aprendizaje que promueva el potencial de los estudiantes?

- Promover la dimensión equitativa de las LLP, incluyendo los temas de abandono escolar prematuro, estudiantes inmigrantes, necesidades educativas especiales y una alta calidad profesional del personal docente y de la directiva escolar. En consecuencia, desde este punto de vista, la cuestión principal que debe hacerse sobre los PSE es: ¿están desfavorecidos o discriminados algunos miembros de la comunidad escolar?, y ¿qué se puede hacer al respecto a fin de respetar el espíritu de los derechos del niño?

Los Servicios Psicológicos en las escuelas pueden contribuir a las LLP mediante: 
- Un seguimiento crítico de la repercusión de las LLP en los estudiantes como aprendices de por vida basada en la Convención de los Derechos del Niño. Aspectos implicados: los derechos humanos, respetando la educación y las escuelas; el desarrollo completo de los potenciales de cada alumno; la autonomía responsable y las prácticas democráticas; el acceso y uso de la información; el respeto del punto de vista del niño o adolescente; la compatibilidad entre la teoría y la práctica educativas; respeto del ocio y el juego; que el trabajo sea significativo; la asistencia escolar; la disciplina y la dignidad humana, y el acceso a asesoramiento y servicios de salud.

- Apoyar el traspaso al sistema educativo de los derechos generales del niño basados en los principios de formación permanente mediante la determinación de la naturaleza y los parámetros de potencial de desarrollo físico, mental, espiritual, moral y social. Esto incluye la ampliación del conocimiento básico de las consecuencias de las teorías educativas y psicológicas, y la aplicación de los conocimientos a la educación y a la práctica psicológica en las escuelas para la consecución de los parámetros de formación permanente.

- Mejorar, implementar y evaluar la orientación permanente y el asesoramiento durante el período de desarrollo más importante de los ciudadanos europeos, entre ellos: el establecimiento y la evaluación de un enfoque coordinado entre los servicios de salud, los sociales y los educativos con el fin de mejorar el desempeño del estudiante y las transiciones que se dan en la educación (de preescolar a escuela, de primaria a secundaria, de obligatoria a formación profesional y educación superior), la identificación precoz y el tratamiento de los obstáculos para un adecuado rendimiento académico, el desarrollo de habilidades/aptitudes vitales con el fin de prevenir la aparición de dificultades de aprendizaje y/o comportamiento, y la promoción de ambientes escolares saludables.

- Centrarse en la formación del profesorado y el asesoramiento en los planes de desarrollo de las escuelas.

- Apoyo a la implementación y evaluación intersectorial de las LLP. Una de las principales competencias especiales del PSE -en comparación con otras profesiones en el ámbito educativo- es su capacidad para evaluar los avances en la investigación educativa y psicológica y su relevancia práctica, debido a su sólida formación metodológica. El PSE puede desempeñar un importante papel en la evaluación de la aplicación de las estrategias de formación permanente y puede poner en práctica los resultados en el triángulo de conocimiento formado por investigación, política y práctica. El PSE puede actuar como un sistema de alerta para los Ministerios de Educación sobre la eficacia de las regulaciones que se lleven a cabo en el sistema educativo.

Afirmación 2. Sólo las estructuras de apoyo escolar, como los Servicios Psicológicos, basadas en datos objetivos, válidos y fiables, pueden promover una aplicación sostenible y exitosa de LLP en toda Europa.

La consabida falta de datos acerca de las estructuras de apoyo escolar en Europa debe ser abordada con el fin de instar a los políticos a tomar decisiones basadas en la evidencia sobre las reformas educativas, como, por ejemplo, las políticas de formación permanente.

Afirmación 3. La inversión en la integración de infraestructuras profesionales de apoyo a las escuelas en Europa significa inversión en juventud y es una manera inteligente de aumentar la eficacia y la eficiencia de nuestra sociedad.

La prevención primaria sigue siendo un área olvidada en la atención sanitaria y en los sistemas educativos. Desde el punto de vista del desarrollo demográfico, con el aumento de los costes sociales y sanitarios para la sociedad, la inversión en prevención primaria en forma de servicios de apoyo escolares reduce los costes en lugar de representar una carga financiera.

La inversión en Servicios Psicológicos Escolares, junto con:

- coordinar la posición de un enfoque integrado intersectorial de apoyo a la escuela para ayudar en la lucha contra las desigualdades de salud, sociales y educativas en la juventud;

- facilitar el papel en el sistema educativo para la promoción de la salud y la prevención del fracaso y/o disfunciones (identificación, derivación, intervención precoz);

- y una posición clave en el apoyo a la aplicación y evaluación de educación, salud y políticas sociales en el sistema educativo.

Todo ello representa una estrategia rentable para mejorar los recursos en educación, salud y sistemas sociales, así como el ahorro de costes importantes evitables a largo plazo para la sociedad.

\section{Conclusiones del Proyecto ESPIL}

\section{Recomendaciones}

El proyecto ESPIL finaliza con una serie de recomendaciones con el fin de adaptar la profesión del psicólogo educativo a estas necesidades (afirmaciones anteriores): 
- Centrarse en las intervenciones de base empírica, basadas en la evidencia.

- Centrarse en la prevención e intervención (la promoción de la salud, incluyendo entornos escolares saludables, prevención de la violencia, intervención en crisis y formación del profesorado), con menos evaluaciones individuales en favor de medidas de prevención a mayor escala.

- Centrarse en los resultados para los niños, familias y escuelas.

- Centrarse en el «bienestar»y la «salud mental» en el sentido de la psicología positiva.

- Enfatizar el uso ético y eficaz de las TIC.

- Defensa de los Derechos del Niño por parte tanto de los individuos como de las comunidades para mejorar su salud, bienestar y rendimiento escolar.

- Desarrollo de planes de estudio basados en competencias profesionales adaptadas a las necesidades de los PSE, como la prevención, los cambios organizativos, las teorías ecológicas de sistemas, el desarrollo, la implementación y evaluación de las intervenciones, la diversidad o el trabajo en equipo multisectorial.

- Hacer hincapié en las relaciones significativas entre la valoración y la intervención, en el sentido de una evaluación inclusiva y un enfoque participativo.

- Hacer hincapié en los enfoques efectivos para la formación permanente de los PSE.

- Aumentar las reflexiones sobre la organización y el trabajo de los PSE.

- Hacer hincapié en la cooperación entre profesionales e investigadores.

- Trabajar en colaboración y crear redes entre disciplinas, sectores y el resto de interlocutores asociados para aumentar el impacto y la sostenibilidad de los programas y las políticas de promoción de la salud y mejorar los logros educativos.

- Mejorar la cooperación europea entre los psicólogos en el sistema educativo.

\section{Documento de la postura de EFPA sobre los psicó- logos en el sistema educativo y su contribución a la formación permanente}

El Consejo Ejecutivo de EFPA redactó un documento sobre la postura que adopta en este tema tras el estudio llevado a cabo mediante el Proyecto ESPIL. Dada la suma importancia del reconocimiento formal e institucional de nuestro estudio, hemos creído relevante finalizar este escrito con las principales reflexiones anotadas en dicho documento.

\section{Psicólogos en el sistema educativo}

Los psicólogos en el sistema educativo son psicólogos profesionales con un máster en psicología educati- va y experiencia en el campo de la educación. La mayoría de ellos son psicólogos que trabajan en ed. infantil, primaria o secundaria, o bien en entornos relacionados con la escuela. Cubren un amplio rango de estudiantes, con edades que van de los 2 a los 20 años. Al tratar con niños en las primeras etapas de su desarrollo, en el entorno cotidiano de la escuela, los psicólogos están en una posición privilegiada para ayudar a cada niño en su desarrollo. Sus principales actividades son:

- Ayudar a los niños a adaptarse al ambiente escolar en las diferentes etapas de su desarrollo.

- Ayudar a la creación de una actitud positiva hacia la escuela y la educación, lo cual proporciona un terreno fértil para el aprendizaje.

- Ayudar a los estudiantes a desarrollar un estilo de vida equilibrado desde una edad temprana.

- Identificar los problemas de comportamiento.

- Identificar las dificultades de aprendizaje, ya sean de carácter general (p. ej., un déficit en las aptitudes cognitivas) o de naturaleza específica (p. ej., la dislexia o el TDAH), ayudar a diseñar programas adecuados de educación y ayudar a los niños a hacer frente a estas dificultades lo antes posible para lograr adquirir todo su potencial y evitar una actitud negativa hacia la educación.

- Ayudar a prevenir e identificar los trastornos conductuales y de salud mental.

- Identificar los déficit en habilidades sociales e intervenir para ayudar a los estudiantes desde una edad temprana.

- Ayudar cuando las dificultades derivadas de privación social, discriminación o familiares estén obstaculizando el progreso del niño. Así mismo, deberá identificar estas dificultades.

El trabajo de los psicólogos en el sistema educativo no se limita a los niños, sino que también incluye a los estudiantes, y se extiende a todas aquellas actividades relacionadas con el sistema educativo (escuela, universidad, comunidad...) y con las diversas estructuras de la sociedad. Sus funciones abarcan la prevención, evaluación e intervención en cada uno de estos tres niveles: el niño o estudiante, las instituciones educativas y la sociedad (recomendamos ver la tabla 5 del artículo aparecido en esta misma revista de Psicología Educativa, vol. 17, n. ${ }^{\circ}$ 1, 2011, pág. 79 , escrito por José A. León y titulado «El psicólogo educativo en Europa», para una mejor comprensión de este modelo de perfil profesional del psicólogo educativo visto como un proceso dinámico, pues está extraído del Proyecto ESPIL).

\section{Contribuciones a la formación permanente}

Los psicólogos en el sistema educativo desempeñan un papel importante y vital en la ejecución de las políticas de formación permanente. Contribuyen a la promoción, implementación y evaluación de la formación perma- 
nente de numerosas maneras. En primer lugar, ofrecen orientación y asesoramiento a los ciudadanos europeos en la parte fundamental de formación de su vida:

- Se ayuda en la formación de actitudes positivas hacia el aprendizaje y se trabaja con niños y adolescentes cuya actitud hacia el aprendizaje no es tan positiva.

- Se ayuda en la rehabilitación de los aprendizajes, para que los adultos aprendan lo que no han aprendido en la escuela (alfabetización, etc.) y aprendan nuevas habilidades necesarias en las sociedades que están experimentando rápidos cambios tecnológicos, sociales y económicos.

- Se ayuda en el asesoramiento de aquellos que tuvieron una escolarización incompleta debido a dificultades específicas de aprendizaje y requieren formación.

- Se ofrece asesoramiento sobre cuestiones pedagógicas.

Además de esto:

- Se ofrece formación al profesorado y asesoramiento sobre la escuela sistémica (inclusiva), y se ayuda en la elaboración de planes de desarrollo de la escuela y de mejora del liderazgo escolar.

- Se apoya la implementación y evaluación de políticas intersectoriales de formación permanente en el sistema educativo y se proporciona información de los resultados del «triángulo del conocimiento», consistente en la investigación, la política y la práctica, lo que genera información valiosa para los responsables de las decisiones gubernamentales del ámbito educativo.

\section{Desarrollo de la psicología en el sistema educativo}

\section{1. ¿Dónde estamos ahora?}

En Europa existe actualmente una gran diversidad entre los países con respecto a:

- La organización de los servicios psicológicos en el sistema educativo.

- Las condiciones de trabajo de los psicólogos en el sistema educativo.

- Las funciones y las prácticas realizadas por los psicólogos en el sistema educativo.

- La situación jurídica de los psicólogos en el sistema educativo.

- La proporción de psicólogos por estudiantes y profesores.

- La disponibilidad de instrumentos de evaluación adecuados.

- La formación básica de los psicólogos en el sistema educativo.

- Oportunidades de desarrollo profesional continuo para los psicólogos en el sistema educativo.

- La organización de la práctica supervisada.

\section{2. ¿Qué nos proponemos?}

3.2.1. Desarrollo de una normativa europea para los servicios de psicología en el sistema educativo. Esta normativa deberá incluir:

- Normas sobre el tipo y la calidad de los servicios ofrecidos.

- Normas sobre procedimientos y mejores prácticas en Europa.

- Normas sobre la proporción de estudiantes por psicólogo.

- Normas para disponer de herramientas de evaluación equivalentes en toda Europa.

3.2.2. Desarrollo de una estructura de apoyo integral. Dicha estructura deberá prever la coordinación de las actividades de los psicólogos en el sistema educativo con los de otros profesionales, así como las actividades de salud y servicios sociales, en el mejor interés de los estudiantes tanto a corto como a largo plazo, es decir, como estudiantes de por vida.

3.2.3. Elaboración de normas de calidad europea para la educación y la formación de psicólogos en el Sistema Educativo, como parte de EuroPsy:

- EFPA ha desarrollado el «Certificado Europeo de Psicología EuroPsy», un punto de referencia común de estándares mínimos de calidad en la educación y la formación de los psicólogos europeos, incluyendo cinco años de formación académica y un año de práctica supervisada, así como la participación regular en el desarrollo profesional continuo (CPD o continuous professional development). EuroPsy responde a las peticiones de las políticas europeas sobre el reconocimiento, la transparencia y la comparación de las cualificaciones profesionales y la movilidad de la mano de obra laboral.

- Esta referencia se debe aplicar a la especialización de psicólogos en el sistema educativo.

\section{Recomendaciones para la acción}

Sobre la base de lo anteriormente expuesto, EFPA recomienda:

- Iniciar proyectos en la UE para ayudar a los psicólogos en el sistema educativo de toda Europa a identificar y difundir las mejores prácticas.

- Iniciar proyectos en la UE que faciliten el aprendizaje con pares, la observación de profesionales y las visitas de estudio.

- Crear un grupo de estudio de la UE para hacer un inventario de las herramientas de evaluación adecuadas para los servicios psicológicos en el sistema educativo, para traducir y adaptar estas herramientas, y para desarrollar futuras herramientas de evaluación.

- Crear un grupo de expertos de la UE (Eurydice) para evaluar y mejorar el conocimiento sobre los 
datos relativos a los Servicios Psicológicos en el Sistema Educativo.

- Establecer un proyecto piloto (como una Red de Apoyo Escolar de la UE o UE School SUN) para promover la innovación y soluciones basadas en la evidencia y sostenibles para apoyar a las escuelas.

- Promover el desarrollo e implementación de un Certificado EuroPsy especializado para los Psicólogos en el Sistema Educativo.

- Proporcionar orientación y apoyo para la fase inicial de los Psicólogos en el Sistema Educativo.

- Establecer un Centro Europeo de Formación Profesional y de Recursos para los Psicólogos en el Sistema Educativo.

\section{Conclusiones}

Todas las crisis demandan con fuerza nuevas y más efectivas respuestas a los viejos problemas. El sistema educativo no es una excepción. Gracias al Proyecto ESPIL es ahora de ámbito público -por si alguien todavía tuviera alguna duda- que incluir a los psicólogos dentro del sistema educativo $-\mathrm{y}$, evidentemente, tanto en primaria como en secundaria y bachilleratosignifica una excelente inversión en lo que respecta a una relación coste-beneficio tanto a corto como a largo plazo, así como una certificación del papel central que desempeña el psicólogo educativo como mediador entre los sectores escolar, social, legal y sanitario.

Tras esta exposición sobre el estado de la psicología educativa en el ámbito internacional -especialmente en Europa-, es nuestro deseo que se haya llegado a un punto de vista más claro y una visión de conjunto más comprehensiva concerniente a dónde estamos y cómo hemos llegado, pero también con respecto a qué cosas nos quedan todavía por hacer, pues es evidente que sin una regulación normativa sobre cuál es la función-perfil-rol del psicólogo educativo y cómo acceder a un puesto de trabajo acorde con ella, poco conseguiremos.
Los pasos dados en muchos países de Europa ya están encaminados en esta misma dirección: definición del perfil, definición del puesto de trabajo, condiciones de acceso, así como para la profesionalización de los psicólogos educativos. Además, hemos visto cómo, en los aspectos básicos, estamos -podríamos decir- que «universalmente» de acuerdo (por lo menos, norteamericanos y europeos, pero también buena parte de asiáticos, sobre todo los de aquellos países que forman parte de ISPA), de manera que parece ser que el trabajo que nos queda en la actualidad en España es básicamente de ámbito político-legal.

Por ello, insto desde mi posición como asesor del Área de Psicología Educativa del CGCOP y como representante en España de NEPES a ejercer una presión incluso mayor de la que estamos realizando en estos momentos para que, de una vez por todas, se reconozca y respete legalmente -pues de manera individual creo que nadie tiene ninguna duda sobre el trabajo que los psicólogos ya estamos realizando dentro de los centros educativos- nuestra profesión y se nos permita trabajar con plenas garantías y en condiciones dignas. El resto del mundo occidental ya se ha puesto las pilas. Por nuestra parte, tanto asociaciones de padres como de directivos de centros educativos, como la Conferencia de Decanos de Facultades de Psicología, asociaciones de psicólogos educativos y el propio Consejo General de Colegios Oficiales de Psicólogos de España -es decir, la mayoría de agentes sociales implicados en nuestra profesión- ya se han manifestado a favor de esta necesidad de reconocimiento legal. La pregunta es, pues, clara: ¿para cuándo los políticos se harán eco de este clamor social y se pondrán también las pilas? Como decía J. F. Kennedy:

«A la larga, sólo hay una cosa más costosa que la educación: no educar.»

( There is only one thing in the long run more expensive than education: no education.»)

\section{Extended Summary}

All crises demand new and more effective responses to old problems. The education system is no exception. The fact that the ESPIL project is now public domain -available for consultation, for example, by psychologists in the educational system, be it in primary or secondary school- means an excellent investment in terms of cost-benefit in both the short and long term, as well as certification of the central role played by the educational psychologist as a mediator between the schools, social, legal and health sectors.

After this discussion on the status of educational psychology at the international level, especially in Europe, we hope to have provided a clearer view and a more comprehensive overview concerning where we are and how we have reached this point, and also what still needs to be done. It is clear that without rules regulating the function-profile-role of the educational psychologists and how to get a job in accordance with this, little will be achieved.

The steps taken in many European countries are already leading in this direction: defining the profile of the job and its access conditions, as well as the professionalization of educational psychologists. We have also seen how, in the basics, we are -we might say- in «universal» agreement (Americans and Europeans, but also much of Asia, especially countries that are part of ISPA), so it seems that the work that we have to do at present in Spain is basically political-legal work.

I therefore urge my colleagues, from my position as adviser of the Educational Psychology Area of the 
CGCOP, and as a representative in Spain of NEPES, to exert even greater pressure than we are doing now, in order to achieve, for once and for all, recognition and legal respect-because I firmly believe that there is now no doubt about the work that psychologists are doing in schools- to our profession and let us work with guarantees and in a dignified way. The rest of the Western world is working on it. For our part, both associations of parents and heads of schools, as well as the Conference of Deans of Faculties of Psychology, associations of educational psychologists and the General Council of Associations of Psychologists of Spain -that is, most social agents involved in our profession- have already spoken in favor of the need for legal recognition. The question then is clear: when will the politicians reflect the social clamor and work in this direction? As J. F. Kennedy said:

"There is only one thing in the long run more expensive than education: no education."

\section{Referencias}

Carreras, L. (2010). Conclusiones del Network of Psychologists in the Educational System (NEPES), grupo de trabajo de la Red de psicólogos educativos de la European Federation of Psychologists' Associations (EFPA). Bratislava, 4 y 5 de diciembre de 2009. Infocop, 19/01/-2010: http://www.infocop.es/view_article.asp? $i$ $d=2721$

Conclusiones del Encuentro Nacional de Profesionales de Psicología de la Educación. Barcelona, 30 y 31 de enero de 2009: http://www.infocop.es/vi-ew_article.asp?id-=2254

EFPA Task Force, Psychologists in the Educational System, Report 2001, consultado en http://www.nepes.eu/?q$=$ node $/ 18$

EFPA POLICY PAPER July 2010, Education, Training and Service of School Psychologists across Europe as an Area of Lifelong Learning. Consultado en: http://www.nepes.eu/files/DRAFT\%20JULY\%202010\%20EFPA\%20POLI CY\%2OPAPER.pdf

León, J.A. (2011). El psicólogo educativo en Europa. Revista de Psicología Educativa, 17, 1, 65-83.

Ministerio de Educación, Propuestas para un Pacto Social y Político por la Educación. Ministerio de Educación. Consultado en: http://www.copbizkaia.org/castellano/ uplo-ads/Informe $\% 20$ P sicologia\%20educativa $\%$ 20 en\%20el\%20Sistema\%20Educativo\%20Espa\%F1ol\% 2015-03-10.pdf)

Manuscrito recibido: 29/07/2011

Revisión recibida: 27/02/2012

Manuscrito aceptado: 08/03/2012 